\title{
POINT TRANSITIVE TRANSFORMATION GROUPS $\left({ }^{(}\right)$
}

\author{
BY \\ ROBERT ELLIS
}

Let $(X, T)$ be a point transitive transformation group (i.e. one in which there exists $x \in X$ with cls $x T=X$ ) with compact phase space $X$ and phase group $T$. Then by [2b] $X$ is a quotient of $(\beta T, T)$ where $\beta T$ is the Stone-Cech compactification of the discrete group $T$. This means that $\mathfrak{e}(X)$ is isomorphic to a $T$-subalgebra (Definition 1$)$ of $\mathfrak{e}(\beta T)$. Moreover, $(X, T)$ may be represented as a transformation group of $T$-homomorphisms of $\mathfrak{e}(X)$ into $\mathfrak{e}(\beta T)$. This suggests the problem of characterizing algebraically various properties of $(X, T)$. For example, it is shown (Proposition 3$)$ that $(X, T)$ is minimal if and only if $\operatorname{ker} \phi=0$ for all the aforementioned homomorphisms $\phi$. Other concepts such as almost periodicity, the enveloping semigroup and the equicontinuous structure relation, relevant to the study of transformation groups are discussed from the algebraic point of view.

When $(X, T)$ is 0 -dimensional, $\mathcal{e}(\beta T)$ may be replaced by $\odot T$, the Boolean algebra of subsets of $T$, and $\mathcal{e}(X)$ by a Boolean $T$-subalgebra $a(X)$ of $\mathcal{P}(T)$. In this case the above proposition takes the form $(X, T)$ is minimal if and only if every element of $a(X)$ is syndetic.

The purpose of this paper is then to show that the collection of point transitive transformation groups may be studied by algebraic and set theoretic methods. It is hoped that this approach will shed some light on old problems and introduce some interesting new ones.

The paper is divided into two sections. $\$ 1$ deals with the general case and $\$ 2$ with the case when $X$ is 0 -dimensional.

Unless specified otherwise, all the transformation groups are assumed to be point transitive with compact phase spaces.

1. Let $T$ be a discrete group, $\beta T$ its Stone-Cech compactification and $\mathfrak{e}$ the space of real-valued continuous functions on $\beta T$ provided with the topology of uniform convergence. Then $[2 \mathrm{~b}](\beta T, T)$ is a point transitive transformation group. For $t \in T, f \in \mathcal{C}$ let $\left[f R_{t}\right]\left[f L_{t}\right]$ denote that element of $\mathfrak{e}$ such that $\left[x\left(f R_{t}\right)=(x t) f\right]\left[x\left(f L_{t}\right)=(t x) f\right](x \in \beta T)$. Then $R_{t} R_{s}=R_{s t}$ and $L_{t} L_{s}$ $=L_{t s}(t, s \in T)$.

Definition 1. Let $a$ be a subalgebra of $\mathcal{C}, I$ an ideal in $a$. Then $[a][I]$ is a [T-subalgebra] [T-ideal] if $\left[f R_{t} \in a\right]\left[f R_{t} \in I\right]$ for all $[f \in a][f \in I]$ and $t \in T$. Let $Q, B$ be two $T$-subalgebras of $\mathcal{C}$ and $\phi$ a homomorphism of $Q$ into $B$.

Received by the editors April 7, 1961.

(') This research was supported by the United States Air Force through the Air Force Office of Scientific Research of the Air Research and Development Command, under Contract AF49(638)-569. Reproduction in whole or in part is permitted for any purpose of the United States Government. 
Then $\phi$ is a $T$-homomorphism if $R_{t} \phi=\phi R_{t}(t \in T)$. Let $a$ be a $T$-subalgebra of e. Then $|a|$ will denote the set of all $T$-homomorphisms of $a$ into $e$. (Note: I assume $1 \in a$ for all subalgebras $a$ of $\mathfrak{C}$ and that $1 \phi=1$ for all homomorphisms $\phi$.)

ReMark 1. For all $t \in T, R_{t}$ and $L_{t}$ are homomorphisms $\mathfrak{C}$ into $\mathfrak{C}$. Moreover $L_{t} \in|\mathfrak{e}|$.

Remark 2. Let $a$ be a $T$-subalgebra of $\mathcal{e}$. Then because every homomorphism is uniformly continuous, cls $Q$ is a $T$-subalgebra and $|a|=\mid$ cls $Q \mid$.

Each $x \in \beta T$ determines a homomorphism [3] of $\beta T$ into $\beta T$ namely $y \rightarrow x y(y \in \beta T)$. Let $L_{x}$ be the map of $\mathfrak{e}$ into $\mathfrak{e}$ determined by $x$. Then $L_{x} \in|\mathfrak{e}|$. In fact

Lemma 1. Let $\phi \in|\mathfrak{e}|$. Then there exists a unique $x \in \beta T$ such that $\phi=L_{x}$.

Proof. The map $f \rightarrow e(f \phi)$ is a homomorphism of $\mathbb{e}$ into $R$. Hence there exists $x \in \beta T$ such that $x f=0$ if and only if $e(f \phi)=0$. This implies that $e\left(f L_{x}\right)=e(f \phi)(f \in \mathcal{C})$. Then $t\left(f L_{x}\right)=e\left(f L_{x} R_{t}\right)=e\left(f R_{t} L_{x}\right)=e\left(f R_{t} \phi\right)=e\left(f \phi R_{t}\right)$ $=t(f \phi)(f \in \mathcal{e}, t \in T)$. Since $f L_{x}$ and $f \phi$ are continuous and $T$ is dense in $\beta T$, $f L_{x}=f \phi(f \in \mathcal{C})$, i.e. $L_{x}=\phi$. The point $x$ is unique since $L_{x}=L_{y}$ if and only if $x=y$.

Lemma 2. Let a be a T-subalgebra of $\mathfrak{e}, \phi \in|\propto|$. Then there exists $\psi \in|\mathfrak{e}|$ with $\psi / Q=\phi$.

Proof. By Remark 2 we may assume that $a$ is uniformly closed. Let $g=[f / f \in Q, e(f \phi)=0]$. Then $g$ is a maximal ideal in $Q$ and so there exists a maximal ideal $\mathfrak{N}$ in $\mathcal{C}$ with $g \subset \mathfrak{N}$. Let $x \in \beta T$ such that $\mathfrak{N}=[g / g \in \mathcal{C}, x g=0]$. Set $\psi=L_{x}$. Then the two homomorphisms $f \rightarrow e(f \phi)$ and $f \rightarrow e(f \psi)(f \in Q)$ determine the same maximal ideal $g$. Hence $e(f \phi)=e(f \psi)(f \in Q)$ and so $f \phi=f \psi(f \in Q)$ as in Lemma 1 since $Q$ is a $T$-subalgebra of $\mathfrak{C}$.

Definition 2. The transformation group $(|Q|, T)$. Let $Q$ be a $T$-subalgebra of $\mathcal{e}, \phi \in|a| t \in T$. Let $\phi t=\phi L_{t}$. Then $\phi e=\phi,(\phi t) s=\phi(t s)(t, s \in T)$; i.e. $T$ acts on $|a|$. The set $|Q|$ can be made into a topological space in several ways. Let $[\Im][\delta]$ be the smallest topology on $|a|$ making the maps $\phi \rightarrow a(f \phi)$ of $|a|$ into $R$ continuous for all $[a \in T][a \in \beta T]$ and $f \in a$. Finally let $u$ be the smallest topology on $|a|$ making the maps $\phi \rightarrow f \phi$ of $|a|$ into $\mathbb{e}$ continuous $(f \in a)$. Then $\Im \subset S \subset \mathcal{U}$. Unless specified otherwise $|a|$ will be assumed provided with the topology 3 .

Proposition 1. Let a be a T-subalgebra of $\mathcal{e}$. Then

(1) The maps $\phi \rightarrow \phi t$ of $|\mathrm{Q}|$ into $|\mathrm{Q}|$ are continuous for all $t \in T$ when $|\mathrm{Q}|$ is provided with any ane of the topologies $\mathrm{J}, \mathrm{S}$, น.

(2) Assume $a \phi \subset a(\phi \in|a|)$. Then

(a) The maps $\phi \rightarrow \psi \phi$ of $|\mathrm{Q}|$ into $|\mathrm{Q}|$ are continuous for all $\psi \in|Q|$ when $|\mathrm{Q}|$ is provided with any one of the topologies $\mathrm{J}, \mathrm{S}$, or $\mathcal{U}$. 
(b) The maps $\phi \rightarrow \phi \psi$ of $|a|$ into $|a|$ are continuous for all $\psi \in|a|$ when $|a|$ is provided with either $\mathfrak{S}$ or $\mathrm{u}$.

(3) The topology $J$ is compact Hausdorff.

(4) The transformation group $(|a|, T)$ is point transitive.

Proof. (1) and (2) follow immediately from the definitions of the terms involved. For (3) consider first the case $a=\mathcal{C}$. Then $L_{x_{\alpha}} \rightarrow L_{x}(\mathfrak{I})$ if and only if $x_{\alpha} \rightarrow x$ in $\beta T$. Hence $|\mathrm{e}|$ is homeomorphic to $\beta T$. Now the map $\phi \rightarrow \phi / a(\phi \in|\mathfrak{e}|)$ of $|\mathfrak{e}|$ onto $|a|$ is a homomorphism onto. Hence $|a|$ is compact and $(|Q|, T)$ is point transitive. Thus (4) is proved. To see that $|a|$ is Hausdorff observe that if $t(f \phi)=t(f \psi)(t \in T, f \in a)$ where $\phi, \psi \in|a|$, then by continuity $x(f \phi)=x(f \psi)(x \in \beta T, f \in Q)$ and so $\phi=\psi$.

COROLlary 1. The proof of 3 shows that $|\mathfrak{e}|$ is isomorphic to $\beta T$.

Lemma 3. Let a be a uniformly closed $T$-subalgebra of $\mathfrak{e}, f \in \mathfrak{C}-a$. Then there exist $\phi, \psi \in \mathcal{C}$ such that $\phi|a=\psi|$ a but $f \phi \neq f \psi$.

Proof. Let $Q$ be the uniformly closed subalgebra of $\mathcal{e}$ generated by $Q$ and $f$. Set $R(Q)=[(x, y) / x, y \in \beta T, x g=y g(g \in Q)]$ and $R(B)=[(x, y) / x, y \in \beta T$, $x g=y g(g \in B)]$. Then $R(B)$ is a closed equivalence relation on $\beta T$ and $\mathbb{B} \cong \mathcal{C}(\beta T / \mathcal{Q}(\mathbb{B}))$. Now $Q$ is a uniformly closed proper subalgebra of $B$. Hence by the Stone-Weierstrass theorem $R(Q) \neq R(B)$. Thus there exist $x, y \in \beta T$ with $x g=y g(g \in Q)$ but $x f \neq y f$. Set $\phi=L_{x}, \psi=L_{y}$.

Proposition 2. Let $Q$, $B$ be two uniformly closed $T$-subalgebras of $\mathfrak{e}$, let $\alpha \in|Q|$. Then

(1) If $Q \alpha \subset \subset, \alpha$ induces a homomorphism $\bar{\alpha}:|\leftrightarrow| \rightarrow|a|$ viz. $\phi \rightarrow \alpha \phi(\phi \in|ß|)$. Conversely, if $\pi:|\Theta| \rightarrow|Q|$ is a homomorphism; then there exists $\alpha \in|\mathrm{Q}|$ with $a \alpha \subset ß$ and $\bar{\alpha}=\pi$.

(2) Let $a \alpha \subset$ B. Then $\alpha$ is [one-one] [onto] [an isomorphism] if and only if $\bar{\alpha}$ is [onto] [one-one] [an isomorphism].

Proof. (1) One verifies directly that $\bar{\alpha}$ is indeed a homomorphism of $|\leftrightarrow|$ into $|Q|$. Now let $\pi$ be a homomorphism of $|ß|$ into $|a|$ and let $\rho:|\mathfrak{e}| \rightarrow|\Theta|$ be the homomorphism of $|\mathfrak{e}|$ into $|\mathbb{B}|$ such that $\phi \rho$ $=\phi / \mathbb{B}(\phi \in|\mathfrak{e}|)$. Set $\alpha=\epsilon \rho \pi$ where $\epsilon$ is the identity on $\mathcal{C}$. Let $\phi \in|\mathfrak{e}|,\left(t_{\beta}\right)=\mathrm{a}$ net on $T$ with $\epsilon t_{\beta} \rightarrow \phi$. Then $\alpha \phi=\lim \alpha t_{\beta}=\lim \epsilon \rho \pi t_{\beta}=\lim \epsilon t_{\beta} \rho \pi=\phi \rho \pi$.

Now let $f \in Q$ and suppose $f \alpha$ were not in $Q$. Then by Lemma 3 there would be $\phi, \psi \in|\mathrm{e}|$ with $\phi \rho=\psi \rho$ and $f \alpha \phi \neq f \alpha \psi$. This would, however, contradict the fact that $f \alpha \phi=f \phi \rho \pi=f \psi \rho \pi=f \alpha \psi$. Hence $Q \alpha \subset \beta$ and $\pi=\bar{\alpha}$. (Notice that $\alpha$ is just the image under $\pi$ of the identity element of $|B|$.)

(2) Suppose $\alpha$ is one-one. Then $D=Q \alpha \subset B$ is a $T$-subalgebra of $a$ and there exists $\psi \in|\mathfrak{e}|$ such that $f \psi \epsilon=f(f \in D)$ and $g \alpha \psi=g(g \in \mathbb{Q})$. This implies that $(\psi \mid A) \bar{\alpha}=\epsilon$, the identity on $|\mathfrak{Q}|$. Since $\epsilon T$ is dense in $|\mathfrak{Q}|,|\Theta|=|\mathfrak{Q}|$.

Conversely suppose $\bar{\alpha}$ is onto. Let $f \in Q$ with $f \alpha=0$. Then $0=f \alpha \phi=f(\phi \bar{\alpha})$ 
$\cdot(\phi \in|ه|)$ whence $f \psi=0(\psi \in|a|)$. Let $x \in \beta T$. Then $0=e\left(f L_{x}\right)=x f$. Hence $f=0$.

Let $\alpha$ be onto, $\phi, \psi \in|ß|$ with $\phi \bar{\alpha}=\psi \bar{\alpha}$. Then $f \alpha \phi=f \alpha \psi(f \in Q)$. Hence $g \phi=g \psi(g \in B)$ and so $\phi=\psi$.

Let $\bar{\alpha}$ be one-one. If there were $f \in B$ and not in $Q \alpha$ there would be $\phi, \psi \in|\mathfrak{e}|$ with $\phi|Q \alpha=\psi| Q \alpha$ and $f \phi \neq f \psi$. This would mean that $(\phi \mid \Theta) \bar{\alpha}$ $=(\psi \mid \Theta) \bar{\alpha}$ but $\phi|\Theta \neq \psi| \Theta$, a contradiction. The proof is completed.

Lemma 4. Let $(X, T),(Y, T)$ be point transitive transformation groups, $\pi$ a homomorphism of $X$ into $Y$, and $\rho, \eta$ homomorphisms of $\beta T$ into $X$ and $Y$ respectively. Then there exists a homomorphism $\psi: \beta T \rightarrow \beta T$ such that $\rho \pi=\psi \eta$.

Proof. Let $e \rho=x \in X$ and $u \eta=x \pi$ for some $u \in \beta T$. Set $v \psi=u v$ [2b] $(v \in \beta T)$. Then $\psi$ is a homomorphism of $\beta T$ into $\beta T$. Moreover $e \rho \pi=x \pi=u \eta$ $=e \psi \eta$ by definition and so $t \rho \pi=t \psi \eta(t \in T)$ since all the maps involved are homomorphisms. Finally $\rho \pi=\psi \eta$ by continuity and the fact that $T$ is dense in $\beta T$.

Proposition 3. Let $(X, T),(Y, T)$ be compact point transitive transformation groups, $\pi$ a homomorphism of $X$ into $Y$. Then there exist $T$-subalgebras $Q, B$ of $\mathcal{C}$, isomorphisms $\rho, \eta$ of $|Q|$ onto $X$ and $|\Theta|$ onto $Y$ and a T-homomorphism $\alpha: \beta \rightarrow Q$ such that $\rho \pi=\bar{\alpha} \eta$ where $\phi \bar{\alpha}=\alpha \phi(\phi \in|Q|)$.

Proof. By Corollary 1 to Proposition 1 and [2b] there exist homomorphisms $\gamma, \delta$ of $|\mathfrak{e}|$ into $X$ and $Y$ respectively. These maps induce homomorphisms $\bar{\gamma}, \bar{\delta}$ of $C(X)$ and $C(Y)$ into e, satisfying the equations $(f \bar{\gamma}) \phi=(\phi \gamma) f$ and $(g \bar{\delta}) \phi=(\phi \delta) g(f \in C(X), g \in C(Y), \phi \in|\mathbb{e}|)$. Let $a=\operatorname{im} \bar{\gamma}, B=\operatorname{im} \delta$. Then $Q$ and $B$ are $T$-subalgebras of $\mathcal{E}$.

Let $\phi_{1}, \phi_{2} \in|\mathfrak{e}|$. Then $\phi_{1} \gamma=\phi_{2} \gamma$ if and only if $\left(\phi_{1} \gamma\right) f=\left(\phi_{2} \gamma\right) f(f \in C(X))$ if and only if $g \phi_{1}=g \phi_{2}(g \in Q)$. I.e., $\phi_{1} \gamma=\phi_{2} \gamma$ iff $\phi_{1} / Q=\phi_{2} / Q$. Thus $\gamma /|a|$ is an isomorphism onto. Similarly $\delta /|\Theta|$ is an isomorphism. Call these isomorphisms $\rho$ and $\eta$ respectively.

By Lemma 4 there exists a homomorphism $\psi:|\mathfrak{e}| \rightarrow|\mathfrak{e}|$ such that $\gamma \pi=\psi \delta$. Let $\phi_{1}, \phi_{2} \in|\mathrm{e}|$ with $\phi_{1} / Q=\phi_{2} / Q$. Then $\phi_{1} \gamma=\phi_{2} \gamma$ whence $\phi_{1} \psi \delta$ $=\phi_{2} \psi \delta$ and so $\phi_{1} \psi / \beta=\phi_{2} \psi / \otimes$. Thus $\psi$ induces a homomorphism $\theta$ of $|a| \rightarrow|\otimes|$ such that $\rho \pi=\theta \delta$. By Proposition 2 there exists $\alpha \in|B|$ with $B \alpha \subset a$ such that $\theta=\bar{\alpha}$. The proof is completed.

In order to extend the results of this paper to more general transformation groups observe that if $(X, T)$ is a transformation group with phase group $T$ and completely regular phase space $X$, then the Stone-Cech compactification $\beta X$ of $X$ may be made into a transformation group with phase group $T$ in a natural fashion, viz., let $t \in T$, then the map $x \rightarrow x T$ of $X$ into $\beta X$ is continuous whence $t$ determines a continuous map $u \rightarrow u t(u \in \beta X)$ of $\beta X$ into $\beta X$. This map will be a homeomorphism onto its inverse being the map determined by $t^{-1}$. The canonical map of $(X, T)$ into $(\beta X, T)$ is then an isomorphism into. 
Definition 3. Let $(X, T)$ be a transformation group with phase group $T$ and completely regular phase space $X$. Then $(X, T)$ is minimal if cls $x T$ $=X(x \in X)$. A point $x \in X$ is an almost periodic point if given any neighborhood $U$ of $X$ there exists a syndetic subset $A$ of $T$ (i.e. a subset $A$ such that $A F=T$ for some finite subset $A$ of $T$ ) with $x A \subset U$.

Proposition 4. Let $(X, T)$ be a point transitive transformation group with completely regular phase space $X$. Then

(1) The transformation group $(\beta X, T)$ is point transitive.

(2) The transformation group $(\beta X, T)$ is minimal if and only if there exists $x \in X$ such that $\operatorname{cls}_{X} x T=X$ and $x$ is an almost periodic point of $(X, T)$.

Proof. (1) Let $x \in X$ with $\operatorname{cls}_{x} x T=X$. Then $\operatorname{cls}_{\beta X} x T=\beta X$.

(2) If $(\beta X, T)$ is minimal, then every point of $\beta X$ is an almost periodic point of $(\beta X, T)$, whence every point of $X$ has the desired property.

Conversely, suppose $x$ is an almost periodic point of $(X, T)$ with cls $x x$ $=X$. Let $u \in \beta X$. If cls $u T \neq \beta X$, then $x \notin$ cls $u T$. Hence there exists a compact neighborhood $U$ of $x$ in $\beta X$ such that $U \cap u T=\varnothing$. Now $U \cap X$ is a neighborhood of $x$ in $X$. Let $A$ be a syndetic subset of $T$ with $x A \subset U$. Then $u \in \beta X$ $=$ cls $x T=$ cls $x A F \subset U F$ since $x A F \subset U F$ which is compact. But this contradicts the fact that $U \cap u T=\varnothing$.

CoROllaRY 1. Let $(X, T)$ be a transformation group with a completely regular phase space $X$, let $x$ be an almost periodic point of $X$. Then (cls $x T, T)$ is minimal.

Definition 4. Let $\left[\mathfrak{J}^{\prime}\right]\left[\mathcal{S}^{\prime}\right]$ be the smallest topology on $\mathrm{e}$ making the functions $f \rightarrow r f$ of $\mathcal{e}$ into $R$ continuous for all $[r \in T][r \in \beta T]$. Then $\mathfrak{J}^{\prime}$ and $\boldsymbol{s}^{\prime}$ are both Hausdorff topologies on $\mathfrak{C}$ with $\mathfrak{J}^{\prime} \subset \mathcal{S}^{\prime}$.

For $f \in \mathfrak{e}, t \in T$ set $f t=f L_{t}$. Then $(f t) s=f(t s)(t, s \in T)$, and $(\mathfrak{C}, T)$ is a transformation group when $\mathcal{C}$ is provided with either of the topologies $\mathfrak{J}^{\prime}$ or $\mathcal{S}^{\prime}$.

Lemma 5. Let $f \in \mathfrak{e}$. Then $\operatorname{cls}_{\mathfrak{J}}^{\prime} f T$ is compact in (e, $\left.\mathfrak{J}^{\prime}\right)$.

Proof. Let $\left(x_{\alpha}\right)$ be a net in $\beta T$. Then $x_{\alpha} \rightarrow x \in \beta T$ if and only if $f L_{x} \rightarrow f L_{x}$ modulo $\mathfrak{J}^{\prime}(f \in \mathcal{C})$. Since $T$ is dense in $\beta T$, this means that cls $\mathfrak{J}^{\prime} f T$ $=\left[f L_{x} \mid x \in \beta T\right]$ and that the map $x \rightarrow f L_{x}$ of $\beta T$ onto $\operatorname{cls}_{\mathfrak{J}}^{\prime} f T$ is a homomorphism onto.

Lemma 6. Let a be a T-subalgebra of $\mathfrak{C}, \phi, \psi \in|a|$. Then $\phi \in \operatorname{cls}_{\mathfrak{J}^{\prime}} \psi T$ if and only if $\operatorname{ker} \psi \subset \operatorname{ker} \phi$.

Proof. Let $\phi \in \operatorname{cls}_{\jmath} \psi T, f \in Q$ with $f \psi=0$. Then there exists a net $\left(t_{\alpha}\right)$ on $T$ such that $t f \psi L_{t_{\alpha}} \rightarrow t f \phi(t \in T)$. But $t f \psi L_{t_{\alpha}}=\left(t_{\alpha} t\right) f \psi=0$. Hence $f \phi=0$.

Now suppose $\operatorname{ker} \psi \subset \operatorname{ker} \phi$. By Remark 2 we may assume $a$ uniformly closed. Then by the Stone-Weierstrass theorem $C(|a|)$ is the collection of functions $\phi \rightarrow t f \phi(t \in T, f \in a)$. Thus if $\phi$ were not in cls $\xi \psi T$ there would be 
$t \in T, f \in Q$ with $t f \psi L_{s}=0(s \in T)$ and $t f \phi \neq 0$. This would mean that $f \psi=0$ but $f \phi \neq 0$, which contradicts the assumption that $\operatorname{ker} \psi \subset \operatorname{ker} \phi$.

Corollary 1. Let a be a T-subalgebra of $\mathfrak{e}, \phi \in|\propto|$. Then $\phi$ is an almost periodic point of $|a|$ if and only if $\operatorname{ker} \phi$ is a maximal proper $t$-ideal of $a$.

Proof. Let $\phi$ be an almost periodic point of $|a|$ and let $g$ be a proper $T$ ideal of $a$ with ker $\phi \subset g$. We may assume that $g$ is a maximal such ideal. Then there exists a maximal proper $T$-ideal $\mathfrak{T}$ of $\mathfrak{C}$ with $\mathfrak{T} \cap a=\mathfrak{g}$ and $\eta \in|\mathfrak{e}|$ with ker $\eta=\mathfrak{T}$. Set $\psi=\eta \mid a$. Then $\operatorname{ker} \phi \subset g=\operatorname{ker} \psi$, whence $\psi \in \operatorname{cls}] \phi T$. Since $\operatorname{cls}_{\mathfrak{J}} \psi T$ is minimal $\phi \in \operatorname{cls}_{\mathfrak{J}} \psi T$. Thus $\mathfrak{g}=\operatorname{ker} \psi \subset \operatorname{ker} \phi$.

Conversely, suppose ker $\phi$ is a maximal proper $T$-ideal of $a$. Let $\psi \in \operatorname{cls}_{\mathfrak{J}} \phi T$. Then $\operatorname{ker} \phi \subset \operatorname{ker} \psi$. Since ker $\psi$ is a proper $T$-ideal of $a$, this means that $\operatorname{ker} \phi=\operatorname{ker} \psi$. Hence $\phi \in \operatorname{cls}_{\mathfrak{J}} \psi T$. The proof is completed.

Proposition 5. Let a be a T-subalgebra of $\mathfrak{C}$. Then the following statements are equivalent:

(1) $\operatorname{ker} \phi=0(\phi \in|a|)$.

(2) $|a|$ is minimal.

(3) $f$ is an almost periodic point of $\left(\mathcal{C}, \boldsymbol{J}^{\prime}\right)$ for all $f \in Q$.

(4) Let $f \in a$ with $f \neq 0$. Then there exists $F$, a finite subset of $T$ such that $\beta T=\bigcup_{t \in \boldsymbol{F}}[x \mid x t f \neq 0]$.

(5) a has no proper nonzero T-ideals.

Proof. (1) implies (2). This is true by Lemma 6. (2) implies (3). Let $f \in a$, $F$ a finite subset of $T$, and $\epsilon>0$. Then $(f, F, \epsilon)=[g /|t f-t g|<\epsilon(t \in F)]$ is a typical neighborhood of $f$ in the topology $J^{\prime}$. Let $\omega$ be the identity element of $|a|$. Then $(\omega, f F, \epsilon)=[\phi / \phi \in|a|,|t f \phi-t f|<\epsilon(t \in F)$ is a neighborhood of $\omega$. Hence there exists a syndetic subset $A$ of $T$ with $\omega s \in(\omega, f F, \epsilon)(s \in A)$. Then $|t(f s)-t f|<\epsilon(t \in F, s \in A)$, i.e. $f$ is an almost periodic point of $\mathfrak{e}$.

(3) implies (4). Let $f \in Q$ with $f \neq 0$. Then there exists $t \in T$ with $t f=c \neq 0$. We may suppose $c>0$. Then there exists a syndetic subset $A$ of $T$ with $f A \subset(f, t, c / 3)$. Let $T=A H$ where $H$ is a finite subset of $T$. If $a \in A$ then $|t(f a)-t f|<c / 3$ and so $a t f \geqq c / 3$. Now set $F=H^{-1} t, E(s)=[x \mid x s f \geqq c / 3]$ and $E=U[E(s) \mid s \in F]$. Then $E$ is a closed subset of $\beta T$. Let $r \in T$. Then $r=a h$ for some $h \in H$. Then $s=h^{-1} t \in F$ and $r s f=a h h^{-1} t f=a t f \geqq c / 3$. Hence $T \subset E$ and so $E=\beta T$.

(4) implies (5). Let $g$ be a nonzero $T$-ideal of $a$. If $g$ were proper there would be $x \in \beta T$ such that $x f=0(f \in \mathfrak{g})$. Let $f \in g$ with $f \neq 0$. Since $f R_{t} \in \mathfrak{g}(t \in \mathfrak{J})$, $0=x\left(f R_{t}\right)=x t f(t \in T)$. But this contradicts (4).

(5) implies (1). This is true because $\operatorname{ker} \phi$ is a proper $T$-ideal for all $\phi \in|a|$. The proof is completed.

Proposition 6. Let $f \in \mathcal{C}$. Then $f$ is an almost periodic point of $\left(\mathfrak{C}, \mathfrak{J}^{\prime}\right)$ if and only if given $\phi \in|\mathfrak{e}|$ there exists $\psi \in|\mathfrak{e}|$ with $f \phi \psi=f$. 
Proof. Let $f$ be an almost periodic point of $\mathfrak{e}, \phi \in|\mathfrak{e}|$. Then $\operatorname{cls}^{\prime} f T$. Hence there exists a net $t_{\alpha}$ of elements of $T$ with $f \phi t_{\alpha} \rightarrow f$ (modulo $J^{\prime}$ ). Let $\psi \in \mid$ e $\mid$ with $L_{\iota_{\alpha} \rightarrow \psi}$ (modulo J). Then $\phi t_{\alpha} \rightarrow \psi$ whence $f \phi \psi=f$.

Let $f \in \mathfrak{C}$ and suppose that given $\phi \in|\mathfrak{e}|$ there exists $\psi \in|\mathfrak{e}|$ with $f \phi \psi=f$. Let $g \in \operatorname{cls}_{\mathfrak{J}^{\prime}} f T$. Then $g=f \phi$ for some $\phi \in|\mathfrak{e}|$. Let $\psi \in|\mathfrak{e}|$ with $f \phi \psi=f$ and $t_{\alpha}$ a net of elements of $T$ with $L_{t_{\alpha}} \rightarrow \psi$. Then $f \phi t_{\alpha} \rightarrow f$, i.e. $f \in \operatorname{cls}^{\prime} g T$. The proof is completed.

COROLlary 1. Let $f$ be an almost periodic point of $\left(\mathfrak{e}, \mathfrak{J}^{\prime}\right)$, a the uniformly closed $T$-subalgebra of $\mathfrak{e}$ generated by $f$. Then $|a|$ is minimal.

Proof. Let $\phi \in|\mathfrak{e}|$. By Proposition 3 and Lemma 2 it suffices to show that $\operatorname{ker} \phi / Q=0$. There exists $\psi \in|\mathfrak{e}|$ with $f \phi \psi=f$. Let $D=[g / g \in \mathfrak{C}, g \phi \psi=g]$. Then $\mathbb{D}$ is a uniformly closed $T$-subalgebra of $\mathcal{e}$ containing $f$. Hence $a \subset D$ and the proof is completed.

Definition 5. Let $X, Y$ be point transitive transformation groups, let $x \in X$ with $\operatorname{cls}(x T)=X$. Then $(X, x)$ covers $Y$ if given $y \in Y$ there exists a homomorphism $\pi: X \rightarrow Y$ with $x \pi=y$. Let $Q$, $\&$ be $T$-subalgebras of $\mathcal{C}$. Then $|a|$ covers $|B|$ if $(|a|, w)$ covers $|\mathbb{B}|$ where $w$ is the identity element of $|a|$.

REMARK 3. Let $X$ be a point transitive transformation group, $E$ its enveloping semigroup, and $e$ the identity element of $E$. Then $(E, e)$ covers $E$.

REMARK 4. Let $X, Y, Z$ be point transitive transformation groups such that $(X, x)$ covers $Y$ and $(Y, y)$ covers $Z$. Then $(X, x)$ covers $Z$.

LEMMA 7. Let $X$ be a point transitive transformation group such that $(X, x)$ covers $X$. Then $(X, x)$ covers the enveloping semigroup $E$ of $X$.

Proof. Let $p, q \in E$ with $x p=x q$, let $y \in X$ and $\pi$ a homomorphism of $X$ into $X$ with $x \pi=y$. Then $y p=x \pi p=x p \pi=x q \pi=x \pi q=y q$. Hence $p=q$. Thus the map of $p \rightarrow x p$ of $E$ into $X$ is an isomorphism. Let $\eta$ denote the inverse map, and let $p \in E$. The map $L_{p}: E \rightarrow E$ such that $q L_{p}=p \Phi(q \in E)$ is a homomorphism of $E$ into $E$ which maps $e$ into $p$. Then $\eta L_{p}$ is a homomorphism of $X$ into $E$ mapping $x$ onto $p$.

LEMMa 8. Let $X, Y$ be point transitive transformation groups, $\pi$ a homomorphism of $X$ onto $Y, E(X), E(Y)$ the enveloping semigroups of $X$ and $Y$. Then $(E(X), e)$ covers $E(Y)$.

Proof. By [3] there exists a homomorphism $\theta: E(X) \rightarrow E(Y)$ onto such that $e \theta=e$. Let $p \in E(Y)$. Then $\theta L_{p}: E(X) \rightarrow E(Y)$ is a homomorphism such that $e \theta L_{p}=p$.

Corollary 1. Let $X, Y$ be point transitive transformation groups, let $(X, x)$ cover $X$ and $Y$. Then $(X, x)$ covers the enveloping semigroup $E(Y)$ of $Y$.

Proof. By Lemma $7(X, x)$ covers the enveloping semigroup $E(X)$ of $X$. 
Now $(E(X), e)$ covers $E(Y)$ by Lemma 8 . Hence $(X, x)$ covers $E(Y)$ by Remark 4.

Lemma 9. Let $Q, B$ be uniformly closed T-subalgebras of $\mathbb{C}$. Then $|a|$ covers $|\otimes|$ if and only if $B \phi \subset Q(\phi \in|\otimes|)$.

Proof. Let $|a| \operatorname{cover}|\otimes|, \phi \in|\otimes|, \pi:|a| \rightarrow|\otimes|$ a homomorphism such that $\omega \pi=\phi$. Then as in the proof of Proposition 2, $\pi=\bar{\phi}$, i.e. $\psi \pi=\phi \psi(\psi \in|a|)$. Then $B \phi \subset a$ by Proposition 2.

Now let $B \phi \subset a(\phi \in|\otimes|)$. Then $\Phi$ is a homomorphism of $|a|$ into $|\otimes|$ such that $\omega \phi=\phi$.

Proposition 7. Let a be a uniformly closed T-subalgebra of $\mathbb{C}, \mathbb{B}$ $=\cup[a \phi|\phi \in| Q \mid]$, and $D$ the uniformly closed T-subalgebra of $\mathbb{e}$ generated by $B$. Then

(1) $B=U\left[\operatorname{cls}_{\jmath^{\prime}} f T / f \in Q\right]$;

(2) $|D|$ is the enveloping semigroup of $|a|$.

Proof. (1) Let $\phi \in|a|, x \in \beta T$ with $\phi=L_{x} \mid A,\left(t_{\alpha}\right)$ a net on $T$ with $t_{\alpha} \rightarrow x$. Then $f t_{\alpha} \rightarrow f \phi\left(\mathcal{J}^{\prime}\right)(f \in Q)$ and so $f \phi \in \operatorname{cls}_{\mathcal{J}^{\prime}} f T(f \in Q, \phi \in|Q|)$. Thus $\operatorname{cls}^{\prime} f T$ $=[f \phi / \phi \in|a|]$.

(2) Let $\phi \in|Q|$. Then $a \phi \subset B \subset D$ and so $|D|$ covers $|a|$. Let $\psi \in|D|$, $f \in B$. Then $f=g \phi$ for some $g \in Q$ and $\phi \in|Q|$. Then $a \phi \subset D$ implies that $\phi \psi \in|a|$. Hence $f \psi=g \phi \psi \in D$. Thus $B \psi C D$ and so $D \psi C D$. Consequently $|D|$ covers $|D|$. Now let $\mathcal{E}$ be a uniformly closed $T$-subalgebra of $\mathcal{C}$ with $|\varepsilon|$ isomorphic to the enveloping semigroup of $|Q|$. Then $|D|$ covers $|\varepsilon|$ and so $\varepsilon \subset D$. Moreover $|\varepsilon|$ covers $|a|$, thus $a \phi \subset \varepsilon(\phi \in|a|)$. I.e. $B \subset \mathcal{E}$ whence $D \subset \varepsilon$.

CoROLlary 1. Let a be a uniformly closed T-subalgebra of $\mathbb{C}$. Then the following statements are equivalent.

(1) $|a|$ covers $|a|$,

(2) $|a|$ is the enveloping semigroup of $|a|$.

(3) $\operatorname{cls}_{\mathfrak{J}^{\prime}} f T \subset a(f \in Q)$.

REMARK 5. Let $a$ be a uniformly closed $T$-subalgebra of $\mathfrak{C}, \mathbb{B}$ $=\left[f / f \in Q, \operatorname{clsg}^{\prime}\left[f R_{t} / t \in T\right]\right.$ compact $], D=\left[f / f \in Q\right.$, cls $\left[f R_{t} / t \in T\right]$ compact $]$. Then $B$ and $D$ are uniformly closed $T$-subalgebras of $a$.

REMARK 6 . The algebras $B$ and $D$ are the weakly almost periodic functions and almost periodic functions respectively of $a$.

Proposition 8. Let a be a uniformly closed T-subalgebra of $\mathfrak{e}, \mathcal{E}$ the $T$ subalgebra corresponding to the enveloping semigroup of $|Q|$. Then the following statements are equivalent.

(1) cls $\left[f R_{t} / t \in T\right]$ is compact $(f \in Q)$. 
(2) $(|a|, T)$ is almost periodic [4].

(3) cls $\left[f R_{t} / t \in T\right]$ is compact $(f \in \mathcal{E})$.

(4) $(|\varepsilon|, T)$ is almost periodic.

(5) $|\mathcal{E}|$ is a compact topological group.

(6) $(|\mathcal{E}|, T)$ is minimal and clss' $\left[f R_{t} / t \in T\right]$ is compact $(f \in \mathcal{E})$.

(7) $(|\mathrm{Q}|, T)$ is minimal and clss' $\left[f R_{t} / t \in T\right]$ is compact $(f \in Q)$.

Proof. Statements (1), (2), (3) are equivalent as are statements (3), (4), (5) by [2a]. Now (3) clearly implies (1). Assume (1). Let $D=[f / f \in \mathcal{E}$, cls $\left[f R_{t} / t \in T\right]$ is compact]. Then $D$ is a uniformly closed $T$-subalgebra of $\mathcal{E}$ such that $D \phi \subset D(\phi \in|D|)$. Since $a \subset D$ this means that $a \phi \subset D(\phi \in|a|)$ whence $|D|$ covers $Q$ and consequently $|D|$ covers $|\mathcal{E}|$. This means that $\mathcal{E} C D$. Hence the first five statements are equivalent.

Clearly (4) implies (6) because $E$ is point transitive; also (6) implies (7). Finally (7) implies (2) [2a].

Corollary 1. Let a be a uniformly closed T-subalgebra of $\mathfrak{e}, S$ the equicontinuous structure relation on $|Q|[3]$. Then $|a| / S \approx|B|$ where $B=[f / f \in Q$, cls $f T$ is compact ].

CoROLlary 2. Let a be a uniformly closed T-subalgebra of $\mathfrak{e}, \mathcal{E}$ the algebra corresponding to the enveloping semigroup of $a, G$ the structure group of $a$ [3]. Then $G \approx|\leftrightarrow|$ where $B=[f / f \in \mathcal{E}$, cls $f T$ is compact $]$.

Remark 7. Let $a=[f / f \in \mathfrak{e}, \operatorname{cls} f T$ is compact $]$. Then $|a|$ is the Bohr compactification of $T$.

Remark 8. Let $Q=\left[f / f \in \mathfrak{e}, \operatorname{clss}^{\prime} f T\right.$ is compact $]$. Then $|a|$ is the weakly almost periodic compactification of $T$ [1].

REMARK 9. Let $a$ be a uniformly closed $T$-subalgebra of $\mathcal{e}, \mathcal{F}$ the collection of finite subsets of $Q$. For $F \in \mathcal{F}$ let $Q(F)$ be the uniformly closed $T$ subalgebra of $\mathfrak{e}$ generated by $F$. If $F, H \in \mathcal{F}$ with $F \subset H$ then $Q(F) \subset Q(H)$ and there is a natural homomorphism of $|Q(H)|$ onto $|Q(F)|$. Thus the family $(|Q(F)| / F \in \mathcal{F})$ forms an inverse system of transformation groups. Also $|a| \approx$ inverse limit $(|a(F)| / F \in \mathcal{F})$ the isomorphism being given by the $\operatorname{map} \phi \rightarrow(\phi / a(F) / F \in \mathcal{F})(\phi \in|a|)$.

2 . In this section the transformation groups $(X, T)$ considered are assumed to be compact point transitive and zero dimensional. Since the topology of $X$ is completely determined by the Boolean algebra of its open-closed subsets, the algebras in this section will be algebras over the two element ring $Z_{2}$ rather than the reals. Thus $\mathcal{C}$ will denote the algebra of continuous $Z_{2}$ valued functions on $\beta T$. Then all the notions and proofs of $\$ 1$, except those involving the general completely regular transformation groups, may be carried over practically verbatim if one substitutes $Z_{2}$ for $R$ and adds the assumption that all transformation groups are zero dimensional. For a general reference see 
[5]. Let us identify $\beta T$ with the set of all ultrafilters on $T$. For $f \in \mathfrak{C}$ set $\operatorname{ch}(f)=[t / t \in T, t f=1]$. Then $c h$ is a bijective map of $\mathbb{e}$ onto $\mathcal{P} T$, the collection of all subsets of $T$. The inverse map is the one which sends $A C P T$ into that element $f$ of $\mathcal{C}$ such that $u f=0$ if $A \notin u$ and $u f=1$ if $A \in u$.

Remark 10. In Definition 4 two topologies $J^{\prime}$ and $\delta^{\prime}$ were defined. The definition of these topologies may be carried over to the present situation by substituting $Z_{2}$ for $R$ in Definition 4. There are thus three topologies on $\mathrm{e}$ or $P T$, the topology of uniform convergence, $J^{\prime}$ and $s^{\prime}$. The topology of uniform convergence is discrete and $J^{\prime}$ is the cartesian product topology $Z_{2}^{T}$. Thus $\left(\mathfrak{C}, \mathfrak{J}^{\prime}\right)$ is compact.

Let $f \in \mathfrak{e}$. Then $(f, F)=[\mathrm{g} / \mathrm{g} \in \mathfrak{e}, t f=t g(t \in F)]$ is a typical neighborhood of $f$ in $\left[\mathfrak{J}^{\prime}\right]\left[\boldsymbol{s}^{\prime}\right]$ where $F$ is a finite subset of $[T][\beta T]$. When this notion is carried over to $\mathcal{P} T$ by means of $c h$, the following results. Let $A \subset T$. Then a typical neighborhood of $A$ in $J^{\prime}$ is of the form $(A, F)=[B / B C T, t \in A$ if and only if $t \in A(t \in F)]$ where $F$ is a finite subset of $T$ and a typical neighborhood of $A$ in $\mathrm{s}^{\prime}$ is of the form $(A, F)=[A / B \subset T, A \in u$ if and only if $B \in u(u \in F)]$ where now $F$ is a finite subset of $\beta T$.

Definition 6. Let $A \subset P T$. Then $a$ is a subalgebra of $P T$ if $\varnothing \in Q$, $A \in Q$ implies $A^{\prime} \in Q$, and $A, B \in Q$ imply that $A \cup B \in Q$. $Q$ is a $T$-subalgebra if in addition $A t \in Q(A \in Q, t \in T)$.

Let $\phi: Q \rightarrow \odot T$. Then $\phi$ is a homomorphism if $\varnothing \phi=\varnothing,(A \phi)^{\prime}=A^{\prime} \phi(A \in Q)$, and $(A \cup B) \phi=A \phi \cup B \phi(A, B \in \mathrm{Q})$. It is a T-homomorphism if $(A t) \phi$ $=(A \phi) t(A \in Q, t \in T)$.

The group $T$ acts on $|a|$ the collection of all $T$-homomorphisms of $Q$ into $P T$ as follows: let $t \in T, \phi \in|a|$ then $\phi t$ denotes that element of $|a|$ such that $A(\phi t)=\left(t^{-1} A\right) \phi(A \in Q)$.

The proof of the following lemma is straightforward.

Lemma 10. (1) $\operatorname{ch}\left(f R_{t}\right)=\operatorname{ch}(f) t^{-1}(f \in \mathcal{e}, t \in T)$.

(2) $\operatorname{ch}\left(f L_{t}\right)=t^{-1} \operatorname{ch}(f)(f \in \mathbb{e}, t \in T)$.

(3) The map $a \rightarrow c h(a)$ is a bijective map of the set of T-subalgebras of $\mathfrak{C}$ onto the set of $T$-subalgebras of $P T$.

(4) Let a be a T-subalgebra of e. For $\phi \in \mid$ Q $\mid \operatorname{set} A k h(\phi)=\operatorname{ch}\left(\left(c h^{-1} A\right) \phi\right)$ $\cdot(A \in \operatorname{ch}(Q))$. Then $k h(\phi) \in|\operatorname{ch}(Q)|$ and the mapping $k h$ is bijective and $k h(\phi t)$ $=k h(\phi) t(\phi \in|a|, t \in T)$. Thus the maps ch, kh may be used to state all the relevant results of $\S 1$ in terms of $P T$ rather than $\mathbb{C}$.

Proposition 9. For $u \in \beta T$ let $\bar{u}$ be that map of $P T$ into $P T$ such that $A \bar{u}=\left[t / A t^{-1} \in u\right]$. Then $\bar{u} \in|\rho T|$ and the map $u \rightarrow \bar{u}(u \in \beta T)$ is an isomorphism.

Proof. By Proposition 1 suitably modified the map $u \rightarrow L_{u}(u \in \beta T)$ is an isomorphism of $\beta T$ onto $|\mathfrak{e}|$. The proof will be completed by showing that 
$k h\left(L_{u}\right)=\bar{u}$. Let $A \subset T, f=c h^{-1} A$. Then $A k h\left(L_{u}\right)=\operatorname{ch}\left(f L_{u}\right)$, and $t \in \operatorname{ch}\left(f L_{u}\right)$ iff (if and only if) $t f L_{u}=1$ iff $(u t) f=1$ iff $A \in u t$ iff $A t^{-1} \in u$; i.e. $A k l\left(L_{u}\right)=A \bar{u}$.

Proposition 10. Let a be a T-subalgebra of $P T$. Then $|a|$ is minimal iff $A$ is syndetic $(\varnothing \neq A \in Q)$.

Proof. In the present context Proposition 3 Statements (1) and (2) read $|a|$ is minimal iff $A \phi=\varnothing$ implies $A=\varnothing(A \in a, \phi \in|a|)$.

Thus suppose $A$ syndetic and $\phi \in|Q|$. Then there exists $F$ a finite subset of $T$ with $A F=T$. Hence $T=T \phi=A \phi F$ whence $A \phi \neq \varnothing$.

Conversely suppose $|a|$ minimal and $\varnothing \neq A \in Q$. If $A$ were not syndetic, the collection $\mathcal{F}=\left[A^{\prime} t / t \in T\right]$ would have the finite intersection property. Then if $u \in \beta T$ with $\mathcal{F} \subset u, A^{\prime} \bar{u}=T$ and $A \bar{u}=\varnothing$ contradicting the minimality of $|a|$.

Proposition 11. Let a be a T-subalgebra of PT. Then the following statements are equivalent.

(1) $(|a|, T)$ is almost periodic.

(2) For each $A \in Q$ there exists a finite subset $F$ of $T$ such that given $t \in T$ there is $s \in F$ with $A t=A s$.

(3) For each $A \in Q$ there exists a syndetic subgroup $H$, a finite subset $K$ of $T$, such that $r H K=A$ and $r H \in A$. Actually $H=\left[t / t r^{-1} A=r^{-1} A\right]$.

Proof. Since the topology of uniform convergence is discrete, Statement 2 is a restatement of Statement 1 of Proposition 6. Hence (1) and (2) are equivalent.

Assume (3). Let $N=$ index of $H$ and $p=$ cardinal of $K$. Then there are only $C_{n, r}$ possibilities for the collection $A t$ for $t \in T$. Hence (3) implies (2).

Assume (2). We may also assume that $e \in F$. Let $F_{1}$ be a maximal subset of $F$ with respect to the properties $e \in F_{1}$ and $B=\bigcap\left[A s / s \in F_{1}\right] \neq \varnothing$. Then $B \cap A t=\varnothing$ or $B \subset A t(t \in T)$. Also $\varnothing \neq B \in Q$ and $|a|$ minimal implies that $B$ is syndetic by Proposition 9.

There are two cases to consider $e \in B$ and $e \notin B$. Let us assume first that $e \in B$. Then $B \subset A s\left(s \in F_{1}\right)$ implies that $s^{-1} \in A\left(s \in F_{1}\right)$. Let $t \in B, s \in F_{1}$. Then $B \cap A s t \neq \varnothing$, whence $B \subset A$ st. Thus $t^{-1} \in A s\left(s \in F_{1}\right)$, i.e. $B^{-1} \subset B$.

Let $H=[t / t A=A]$, let $t \in H$. Since $s^{-1} \in A\left(s \in F_{1}\right), t \in A s\left(s \in F_{1}\right)$; i.e. $t \in B$. Now let $b \in B, a \in A$. Then $B \cap A a^{-1} b \neq \varnothing$; therefore $B \subset A a^{-1} b$ and so $b^{-1} a \in A$. Thus $b^{-1} A \subset A(b \in B)$. Since $B^{-1} \subset B$, this means that $b A=A(b \in B)$. Consequently $B=H$.

Since $H$ is syndetic there exists $L$ finite with $H L=T$. Set $K=[1 / 1 \in L$, $H 1 \cap A \neq \varnothing]$. Then of course $A \subset H K$. Let $k \in K, h \in H$. Then there exists $m \in H$ with $m k \in A$. Then $h k=h m^{-1} m k \in H A \subset A$. Hence $A=H K$.

Finally if $e \notin B$, set $C=b^{-1} A$ where $b \in B$. Then the above argument may be applied verbatim to $C$ and $D$ instead of $B$ and $A$ to show that $C=[t / t D=D]$ 
is a syndetic subgroup of $T$ with $D=C K$ for some finite set $K$. Then $A=b C k$ and $C=\left[t / t b^{-1} A=b^{-1} A\right]$. The proof is completed.

In general $H$ need not be in $a$. However, $H$ always belongs to $\&$ the $T$ subalgebra of $P T$ with $|\varepsilon|$ the enveloping semigroup of $|a|$.

\section{REFERENCES}

1. Karel deLeeuw and Irving Glicksberg, Almost periodic compactifications, Applied Mathematics and Statistics Laboratory, Stanford University Technical Note No. 1, December, 1958.

2a. Robert Ellis, Equicontinuity and almost periodic functions, Proc. Amer. Math. Soc. vol. 10 (1959) pp. 637-643.

2b. - Universal minimal sets, Proc. Amer. Math. Soc. vol. 11 (1960) pp. 540-543.

3. Robert Ellis and W. H. Gottschalk, Homomorphisms of transformation groups, Trans. Amer. Math. Soc. vol. 94 (1960) pp. 258-271.

4. W. H. Gottschalk and G. A. Hedlund, Topological dynamics, Amer. Math. Soc. Colloquium Publications vol. 36, 1955.

5. Paul Halmos, Boolean algebra, University of Chicago (notes).

University of Pennsylvania,

Philadelphia, Pennsylvania 